\title{
A Remark on Torsion Euler Classes of Circle Bundles
}

\author{
Shigeaki MIYOSHI \\ Komazawa University \\ (Communicated by K. Kobayasi)
}

\begin{abstract}
We show that any torsion class $e \in H^{2}(M ; Z)$ of any closed manifold $M$ is realized as the Euler class of a smoothly foliated orientable circle bundle over $M$. In the case where $M$ is a 3-manifold, we construct the homomorphism $\pi_{1}(M) \rightarrow S O(2) \subset \operatorname{Diff}_{+}^{\infty}\left(S^{1}\right)$ explicitly whose Euler class is the given torsion class.
\end{abstract}

\section{Introduction and statement of the result.}

Let $M$ be a closed orientable manifold and $\xi=\{E \rightarrow M\}$ an orientable circle bundle over $M$. We denote by $e(\xi)$ the Euler class of $\xi$. As is well known, orientable circle bundles are classified by their Euler classes. On the other hand, foliated orientable circle bundles are classified by their total holonomy homomorphisms. Namely, there is a natural bijection between the set of all smoothly $\left(C^{\infty}\right)$ foliated orientable circle bundles over $M$ modulo leaf preserving bundle isomorphism and the set of all homomorphisms $\pi_{1}(M) \rightarrow \operatorname{Diff}_{+}^{\infty}\left(S^{1}\right)$ modulo conjugacy (cf. $[\mathrm{HH}])$. Here, Diff.$+\left(S^{1}\right)$ denotes the group of all orientation preserving diffeomorphisms of the circle. We consider a homomorphism $\pi_{1}(M) \rightarrow \operatorname{Diff}_{+}^{\infty}\left(S^{1}\right)$ as an equivalent of a smoothly foliated orientable circle bundle over $\boldsymbol{M}$.

In [My] we studied the problem of the existence of a codimension-one foliation transverse to the fibers of a given circle bundle $E \rightarrow M$, that is, the question when is a circle bundle foliated, in the case where the base space $M$ is a 3-manifold. In case the base space is a surface $M=\Sigma$, the necessary and sufficient condition for the existence of a transverse foliation was obtained by J. Milnor and J. W. Wood in [M] and [W]. Assume $\Sigma$ is connected. Denote by $\chi(\xi)$ the Euler number of the circle bundle $\xi$ and set $\chi_{-}(\Sigma)=\max \{0,-\chi(\Sigma)\}$, where $\chi(\Sigma)$ denotes the Euler characteristic of $\Sigma$. Then, there exists a transverse foliation if and only if $|\chi(\xi)| \leq \chi_{-}(\Sigma)$. Here, $\Sigma$ is a closed orientable surface and we omit the non-orientable case for simplicity. We call this inequality Milnor-Wood inequality. In higher dimensions, Milnor-Wood inequality induces a necessary condition for the existence as follows (cf. [M], [W], [My]): If there exists a transverse foliation on the total space $E$, then the following condition is satisfied:

(MW) : $|\langle e(\xi), z\rangle| \leq x(z)$ for any $z \in H_{2}(M ; \mathbf{Z})$.

Received December 8, 1999

Revised May 24, 2000 
Here, $\langle$,$\rangle denotes Kronecker product and x$ is Thurston norm, that is, the pseudonorm on $H_{2}(M ; Z)$ defined as follows: for any $z \in H_{2}(M ; Z), x(z)$ is defined to be the minimum $\chi_{-}(\Sigma)$ of all surfaces $\Sigma$ in $M$ each of which represents the given homology class $z$ (cf. [Th]). In case $\Sigma$ is not connected, here, we set $\chi_{-}(\Sigma)=\sum_{i} \max \left\{0, \chi_{-}\left(\Sigma_{i}\right)\right\}$ with respect to the decomposition into connected components $\Sigma=\bigsqcup_{i} \Sigma_{i}$.

We showed in [My] there exists a family of circle bundles each of which has a transverse foliation of class $C^{0}$ but none of class $C^{3}$. Also we proved with some exceptions the condition (MW) is sufficient for the existence of a $C^{\infty}$ transverse foliation if the base space is a closed Seifert fibred manifold.

In this paper, we consider the case where the condition (MW) is trivial. In fact, we show the following:

THEOREM. Suppose $\xi=\{E \rightarrow M\}$ is an orientable circle bundle over a closed manifold $M$. The dimension of the base space $M$ is arbitrary. If the Euler class $e(\xi)$ is a torsion class in $H^{2}(M ; Z)$, then there exists a codimension-one $C^{\infty}$ foliation on $E$ which is transverse to the fibres. In fact, we can construct the transverse foliation whose total holonomy group is contained in $S O(2)$.

In [M], Milnor showed that the Euler class of a flat $S O(m)$-bundle is a torsion element.

We will prove Theorem in $\$ 2$. In \$3, in connection with the results of the paper [My], we explicitly construct the homomorphism $\pi_{1}(M) \rightarrow S O(2) \subset \operatorname{Diff}_{+}^{\infty}\left(S^{1}\right)$ whose Euler class is the given torsion class if $M$ is a 3-manifold.

\section{Proof of Theorem.}

In this section we prove Theorem. We identify the rotation group $S O(2)$ with $S^{1}=\mathbf{R} / \mathbf{Z}$. Suppose that $M$ is a closed orientable manifold. Consider the short exact sequence:

$$
0 \rightarrow \mathbf{Z} \rightarrow \mathbf{R} \rightarrow \operatorname{SO}(2) \rightarrow 0,
$$

then we have the following exact sequence:

$$
\cdots \rightarrow H^{1}(M ; S O(2)) \stackrel{\beta}{\rightarrow} H^{2}(M ; \mathbf{Z}) \rightarrow H^{2}(M ; \mathbf{R}) \rightarrow H^{2}(M ; S O(2)) \rightarrow \cdots,
$$

where $\beta$ is the Bockstein cohomology homomorphism corresponding to the coefficient sequence above (cf. [S]). By the exactness of the sequence above, we have

$$
\begin{aligned}
\operatorname{Im}(\beta) & =\operatorname{Ker}\left(H^{2}(M ; \mathbf{Z}) \rightarrow H^{2}(M ; \mathbf{R})\right) \\
& =\operatorname{Tor}\left(H^{2}(M ; \mathbf{Z})\right)
\end{aligned}
$$

where Tor denotes the torsion subgroup. On the other hand, any homomorphism $\varphi: \pi_{1}(M) \rightarrow$ $S O(2)$ can be considered as a cohomology class of $H^{1}(M ; S O(2))$ by the universal coefficient theorem

$$
\begin{aligned}
\operatorname{Hom}\left(\pi_{1}(M), S O(2)\right) & \cong \operatorname{Hom}\left(H_{1}(M), S O(2)\right) \\
& \cong H^{1}(M ; S O(2)) .
\end{aligned}
$$


From now on, we identify these groups via these natural isomorphisms. Recall that a homomorphism $\pi_{1}(M) \rightarrow S O(2)$ is considered as an equivalent of a $C^{\infty}$ foliated orientable circle bundle over $M$ whose total holonomy is contained in $S O(2)$. Now we claim the following:

CLAIM (cf. [M]). The Euler class of a homomorphism $\varphi: \pi_{1}(M) \rightarrow S O(2)$ is equal to $-\beta(\varphi) \in H^{2}(M ; \mathbf{Z})$.

PROOF OF CLAIM. First, note that a cochain $C_{1}(M) \rightarrow S O(2)$ is a cocycle if and only if its restriction to the boundaries $B_{1}(M)$ is zero. Suppose a homomorphism $\varphi: H_{1}(M ; Z) \rightarrow$ $S O(2)$ is given. We will define a 1-cocycle which represents $\varphi \in \operatorname{Hom}\left(H_{1}(M ; Z), S O(2)\right) \cong$ $H^{1}(M ; S O(2))$. Since the short exact sequence of 1 -cycles, 1 -chains and 0 -boundaries

$$
0 \rightarrow Z_{1}(M) \rightarrow C_{1}(M) \stackrel{\partial}{\rightarrow} B_{0}(M) \rightarrow 0
$$

is split, we have a direct sum decomposition $C_{1}(M)=Z_{1}(M) \oplus B$, where $B \subset C_{1}(M)$ is a subgroup isomorphic to $B_{0}(M)$. We define a cochain $c: C_{1}(M) \rightarrow S O(2)$ as $\varphi \cdot \pi$ on $Z_{1}(M)$ and 0 on $B$, where $\pi: Z_{1}(M) \rightarrow H_{1}(M ; Z)$ is the natural quotient homomorphism. Then $c$ is a cocycle, that is, $\delta c=0$ and $c$ represents the class $\varphi \in H^{1}(M ; S O(2))$. For, we have $\delta c=c \cdot \partial=\varphi \cdot \pi \cdot \partial=\varphi \cdot \pi \mid B_{1}(M)=0$ and also $c(\zeta)=\varphi \cdot \pi(\zeta)=\varphi[\zeta]$ for any $\zeta \in Z_{1}(M)$.

Next, we show that $-\beta(\varphi)=e(\varphi)$, where $\beta: H^{1}(M ; S O(2)) \rightarrow H^{2}(M ; \mathbf{Z})$ is the Bockstein homomorphism. Indeed, we will show that a representative cocycle of $-\beta(\varphi)$ also represents the primary obstruction class $e(\varphi)$ of the circle bundle defined by $\varphi$. First, the homomorphism $\beta$ is defined through the snake diagram as follows:

$$
\begin{aligned}
& 0 \rightarrow \operatorname{Hom}\left(C_{1}(M), \mathbf{Z}\right) \rightarrow \operatorname{Hom}\left(C_{1}(M), \mathbf{R}\right) \rightarrow \operatorname{Hom}\left(C_{1}(M), S O(2)\right) \rightarrow 0 \\
& \downarrow \partial^{*} \quad \downarrow \partial^{*} \quad \downarrow \partial^{*} \\
& 0 \rightarrow \operatorname{Hom}\left(C_{2}(M), \mathbf{Z}\right) \rightarrow \operatorname{Hom}\left(C_{2}(M), \mathbf{R}\right) \rightarrow \operatorname{Hom}\left(C_{2}(M), S O(2)\right) \rightarrow 0
\end{aligned}
$$

For any 1-cocycle $h \in \operatorname{Hom}\left(C_{1}(M), S O(2)\right)$, there is $\tilde{h} \in \operatorname{Hom}\left(C_{1}(M), \mathbf{R}\right)$ which maps to $h$. Since $\partial^{*} \tilde{h} \in \operatorname{Hom}\left(C_{2}(M), \mathbf{R}\right)$ goes to 0 in $\operatorname{Hom}\left(C_{2}(M), S O(2)\right), \partial^{*} \tilde{h}$, in fact, lies in $\operatorname{Hom}\left(C_{2}(M), \mathbf{Z}\right)$. Denote it by $g \in \operatorname{Hom}\left(C_{2}(M), \mathbf{Z}\right)$. This 2-cochain $g$ is a cocycle and $\beta[h]$ is defined to be the cohomology class of $g$.

Let $f: C_{2}(M) \rightarrow \mathbf{Z}$ be the 2-cocycle defined by chasing the snake diagram from the 1-cocycle $c \in \operatorname{Hom}\left(C_{1}(M), S O(2)\right)$, the representative cocycle of $\varphi$. Thus, $f$ represents $\beta[c]=\beta(\varphi)$. Indeed, the 2-cocycle $f$ is defined as follows: Fix a triangulation of $M$ and suppose $\pi_{1}(M)=\langle G \mid R\rangle$ is the presentation associated with the triangulation of $M$. Namely, each element of $G$ corresponds to an oriented edge which is not contained in a fixed maximal tree and each word of $R$ corresponds to an oriented 2-simplex. From now on we consider each generator $g \in G$ as an edge which is not contained in the maximal tree. Then, by the definition of $c$ we have $c(g)=\varphi(g)$ for $g \in G$. Choose a lift $\widetilde{\varphi(g)} \in \mathbf{R}$ of $\varphi(g) \in S O(2)$ for each $g \in G$. We define a lift $\tilde{c}: C_{1}(M) \rightarrow \mathbf{R}$ of $c: C_{1}(M) \rightarrow S O(2)$ by setting $\tilde{c}(g)=\widetilde{\varphi(g)}$ for each $g \in G$. Note that for an edge contained in the maximal tree the value of $\tilde{c}$ is defined to be zero. By definition, $f=\partial^{*} \tilde{c}$. Now, let $\Delta$ be any oriented 2-simplex and 
suppose its boundary $\partial \Delta$ corresponds to $h_{1}^{\varepsilon_{1}} h_{2}^{\varepsilon_{2}} h_{3}^{\varepsilon_{3}}\left(h_{i} \in G \cup\{1\}, \varepsilon_{i}= \pm 1\right)$. Then since $\partial \Delta$ determines a word consists of the letters of $G$ which belongs to (the normal closure of) $R$, $\varepsilon_{1} \tilde{c}\left(h_{1}\right)+\varepsilon_{2} \tilde{c}\left(h_{2}\right)+\varepsilon_{3} \tilde{c}\left(h_{3}\right)=\varepsilon_{1} \widehat{\varphi\left(h_{1}\right)}+\varepsilon_{2} \widehat{\varphi\left(h_{2}\right)}+\varepsilon_{3} \widehat{\varphi\left(h_{3}\right)}$ is an integer. Thus, we have

$$
\begin{aligned}
f(\Delta) & =\partial^{*} \tilde{c}(\Delta) \\
& =\tilde{c}(\partial \Delta) \\
& =\varepsilon_{1} \tilde{c}\left(h_{1}\right)+\varepsilon_{2} \tilde{c}\left(h_{2}\right)+\varepsilon_{3} \tilde{c}\left(h_{3}\right) \\
& =\varepsilon_{1} \widehat{\varphi\left(h_{1}\right)}+\varepsilon_{2} \widehat{\varphi\left(h_{2}\right)}+\varepsilon_{3} \widehat{\varphi\left(h_{3}\right)} .
\end{aligned}
$$

This implies that $-f$ is the Euler cocycle of $\varphi$. Q.E.D. of Claim.

Recall that $\operatorname{Im}(\beta)$ is the torsion subgroup of $H^{2}(M ; \mathbf{Z})$. Thus, by this Claim the Euler class of a foliated circle bundle whose total holonomy $\pi_{1}(M) \rightarrow S O(2)$ is a torsion class and conversely any torsion class in $H^{2}(M ; \mathbf{Z})$ can be the Euler class of a total holonomy $\pi_{1}(M) \rightarrow S O(2)$. Now the proof is completed.

\section{Explicit construction in dimension three.}

In the case of dimension three we explicitly construct the homomorphism $\pi_{1}(M) \rightarrow$ $\operatorname{Diff}_{+}^{\infty}\left(S^{1}\right)$ which represents the given torsion class. In this section, every coefficient group of homology group is $\mathbf{Z}$.

First, we see how the Euler class of an orientable circle bundle describes the twist of the bundle. Suppose an orientable circle bundle $\xi=\{E \rightarrow M\}$ over a closed 3-manifold $M$ is given. For simplicity we assume $M$ is orientable. We choose an orientation on $M$ and take an oriented embedded loop $K$ in $M$ which represents the Poincare dual of $e(\xi)$. Denote by $\mathcal{E}_{M}(K)$ the exterior of $K$, that is, $\mathcal{E}_{M}(K)=M-\operatorname{int} N(K)$ where $N(K)$ denotes a small tubular neighbourhood of $K$ in $M$. Since $e(\xi) \mid \mathcal{E}_{M}(K)=0$, the restriction $\xi \mid \mathcal{E}_{M}(K)$ is trivial. Fix trivializations $\mathcal{E}_{M}(K) \times S^{1} \cong E \mid \mathcal{E}_{M}(K)$ and $N(K) \times S^{1} \cong E \mid N(K)$. Then the gluing diffeomorphism $g: \partial\left(\mathcal{E}_{M}(K)\right) \times S^{1} \rightarrow \partial N(K) \times S^{1}$ is defined to be the map which makes the following diagram commutative:

$$
\begin{array}{cc}
\partial\left(E \mid \mathcal{E}_{M}(K)\right) & \cong \partial\left(\mathcal{E}_{M}(K)\right) \times S^{1} \\
\| & \downarrow g \\
\partial(E \mid N(K)) & \cong \partial N(K) \times S^{1}
\end{array}
$$

Now fix a framing $S^{1} \times D^{2} \cong N(K)$ so that the gluing diffeomorphism $g$ is represented as a diffeomorphism $S^{1} \times \partial D^{2} \times S^{1} \rightarrow S^{1} \times \partial D^{2} \times S^{1}$, which is expressed as follows:

$$
\left(\begin{array}{lll}
1 & 0 & 0 \\
0 & 1 & 0 \\
m & n & 1
\end{array}\right) \text {. }
$$

Note that on the boundary tori, the framings are the same: $S^{1} \times \partial D^{2} \cong \partial N(K)=\partial\left(\mathcal{E}_{M}(K)\right)$. Here $m$ is an ambiguity of the choices and we can assume $m=0$ by choosing another 
trivialization over $N(K)$. If the Euler class $e(\xi)$ is a torsion element, then the integer $n$ in the above expression is determined modulo the order of $e(\xi)$. Namely, suppose $p e(\xi)=$ $0(p \in \mathbf{Z}, p>0)$ and $q e(\xi) \neq 0$ if $0<q<p(q \in \mathbf{Z})$, then $n$ changes into $n+l p$ $(l \in \mathbf{Z})$ by changing trivialization over $\mathcal{E}_{M}(K)$. Consequently, if the Euler class $e(\xi)$ is a torsion element, then the integer $n$ modulo the order of $e(\xi)$ depends only on the Euler class $e(\xi)$. This representation of the gluing map $g$ implies that the meridian loop of $K$ on the cross section over $\mathcal{E}_{M}(K)$ winds up $n$ times in the fibre direction.

Now we construct the homomorphism. Denote by $\widetilde{S O(2)}$ the universal covering group of $S O(2)$. Recall that we identify $S O(2)$ with $S^{1}=\mathbf{R} / \mathbf{Z}$ and $\widehat{S O(2)}$ with $\mathbf{R}$. It is sufficient for our task that we define a homomorphism from $\pi_{1}\left(\mathcal{E}_{M}(K)\right)$ into $\widehat{S O(2)}$ such that $[\mu]$ is forced to be mapped to the translation by $n$, where $\mu$ denotes the meridian loop of $K$. Then the homomorphism goes down to $\pi_{1}(M)=\pi_{1}\left(\mathcal{E}_{M}(K)\right) /\langle[\mu]\rangle \rightarrow S O(2)$ as desired.

Since $[K] \in H_{1}(M)$ is a torsion element, there is no $z \in H_{2}(M)$ such that the intersection number $[K] \cdot z \neq 0$. Therefore, $H_{2}(M) \rightarrow H_{2}\left(M, \mathcal{E}_{M}(K)\right)$ is the zero map so that $\partial: H_{2}\left(M, \mathcal{E}_{M}(K)\right) \rightarrow H_{1}\left(\mathcal{E}_{M}(K)\right)$ is injective. It is obvious that the meridian loop $\mu$ of $K$ represents an element of infinite order of $H_{1}\left(\mathcal{E}_{M}(K)\right)$.

We will define a homomorphism $\widetilde{\psi}: \pi_{1}\left(\mathcal{E}_{M}(K)\right) \rightarrow \widetilde{S O(2)}$ via $H_{1}\left(\mathcal{E}_{M}(K)\right)$ by choosing a homomorphism $H_{1}\left(\mathcal{E}_{M}(K)\right) \rightarrow \widehat{S O(2)}$ such that $[\mu]$ is forced to be mapped to the translation by $n$. First, we choose a direct sum decomposition of $H_{1}\left(\mathcal{E}_{M}(K)\right)=F \oplus T$, where $T$ is the torsion subgroup and $F$ is a complementary free part. We assume that $[\mu] \in F$ and choose free basis $\alpha_{1}, \cdots, \alpha_{r}$ for the free part $F$. Then, changing signs of $\alpha_{i}$ 's if necessary, we have an expression $[\mu]=\sum_{i=1}^{r} a_{i} \alpha_{i}$ where $a_{i} \in \mathbf{Z}, a_{i} \geq 0$ and $\sum_{i=1}^{r} a_{i} \geq 1$. Note that $[\mu] \neq 0$. Define a homomorphism $\rho: H_{1}\left(\mathcal{E}_{M}(K)\right) \rightarrow \widehat{S O(2)}$ by

$$
\begin{aligned}
\rho\left(\alpha_{i}\right) & =\operatorname{sh}\left(\frac{n}{\sum_{i=1}^{r} a_{i}}\right) \\
\rho \mid T & =\mathrm{id},
\end{aligned}
$$

where $\operatorname{sh}(t)$ denotes the translation by $t$. Composing $\rho$ with the natural quotient homomorphism $\pi_{1}\left(\mathcal{E}_{M}(K)\right) \rightarrow H_{1}\left(\mathcal{E}_{M}(K)\right)$ we have the desired homomorphism. This completes our construction.

ACKNOWLEDGEMENTS. Thanks are due to the referee whose comments improve and simplify the presentation of the paper.

\section{References}

[HH] G. Hector and U. HIRSCH, Introduction to the Geometry of Foliations, Part A, Vieweg (1981).

[M] J. MILNOR, On the existence of a connection with curvature zero, Comment. Math. Helv. 32 (1957-58), 215-223.

[My] S. MiYoshi, On foliated circle bundles over closed orientable 3-manifolds, Comment. Math. Helv. 72 (1997), $400-410$.

[S] E. H. SPANIER, Algebraic Topology, Springer (1995). 
[Th] W. P. ThuRSTON, A norm for the homology of three-manifolds, Mem. A.M.S. 59, No. 339 (1986).

[W] J. W. WoOD, Bundles with totally disconnected structure group, Comment. Math. Helv. 46 (1971), $257-273$.

Present address:

DePaRTMEnT OF MATHEMATICS, ChUo UNIVERSiTy,

KASUGA, BUNKYO, TOKYO, 112-8551 JAPAN.

e-mail: miyoshi@math.chuo-u.ac.jp 